\title{
Effects of practice and experience on the arcuate fasciculus: comparing singers, instrumentalists, and non-musicians
}

\author{
Gus F. Halwani ${ }^{1,2}$, Psyche Loui ${ }^{2}$,Theodor Rüber ${ }^{2,3}$ and Gottfried Schlaug ${ }^{2 *}$ \\ Program in Speech and Hearing Bioscience and Technology, Harvard-MIT Division of Health Sciences and Technology, Massachusetts Institute of Technology, \\ Cambridge, MA, USA \\ 2 Music and Neuroimaging Laboratory, Department of Neurology, Beth Israel Deaconess Medical Center/Harvard Medical School, Boston, MA, USA \\ ${ }^{3}$ Department of Epileptology, Bonn University Hospital, Bonn, Germany
}

\section{Edited by:}

Lutz Jäncke, University of Zurich,

Switzerland

Reviewed by:

Mathias S. Oechslin, University of

Geneva, Switzerland

Jürgen Hänggi, University of Zurich,

Switzerland

\section{*Correspondence:}

Gottfried Schlaug, Department of Neurology, Beth Israel Deaconess Medical Center and Harvard Medical School, 330 Brookline Avenue, Palmer 127, Boston, MA 02215, USA. e-mail: gschlaug@bidmc.harvard.edu
Structure and function of the human brain are affected by training in both linguistic and musical domains. Individuals with intensive vocal musical training provide a useful model for investigating neural adaptations of learning in the vocal-motor domain and can be compared with learning in a more general musical domain. Here we confirm general differences in macrostructure (tract volume) and microstructure (fractional anisotropy, FA) of the arcuate fasciculus (AF), a prominent white-matter tract connecting temporal and frontal brain regions, between singers, instrumentalists, and non-musicians. Both groups of musicians differed from non-musicians in having larger tract volume and higher FA values of the right and left AF. The AF was then subdivided in a dorsal (superior) branch connecting the superior temporal gyrus and the inferior frontal gyrus (STG $\leftrightarrow$ IFG), and ventral (inferior) branch connecting the middle temporal gyrus and the inferior frontal gyrus (MTG $\leftrightarrow$ IFG). Relative to instrumental musicians, singers had a larger tract volume but lower FA values in the left dorsal AF (STG $\leftrightarrow$ IFG), and a similar trend in the left ventral AF (MTG $\leftrightarrow$ IFG). This between-group comparison controls for the general effects of musical training, although FA was still higher in singers compared to non-musicians. Both musician groups had higher tract volumes in the right dorsal and ventral tracts compared to non-musicians, but did not show a significant difference between each other. Furthermore, in the singers' group, FA in the left dorsal branch of the AF was inversely correlated with the number of years of participants' vocal training. Our findings suggest that long-term vocal-motor training might lead to an increase in volume and microstructural complexity of specific whitematter tracts connecting regions that are fundamental to sound perception, production, and its feedforward and feedback control which can be differentiated from a more general musician effect.

Keywords: plasticity, white matter, arcuate fasciculus, auditory-motor interactions, tractography, music, singing

\section{INTRODUCTION}

In recent years, there has been increased interest in the use of musicians to examine brain adaptation in response to intense and longterm training of musical skills (Trainor et al., 1999; Ross et al., 2003; Bengtsson et al., 2005; Koelsch et al., 2005; Zatorre et al., 2007; Hyde et al., 2009; Moreno et al., 2009; Oechslin et al., 2009; Schlaug et al., 2009a; Wan and Schlaug, 2010b). Although most of the previous work has focused on instrumental training, one type of musical training that may provide additional insights in brain adaptation is training in the voice or auditory-vocal domain (Zarate and Zatorre, 2008; Kleber et al., 2010; Zarate et al., 2010). Singing is an intensive auditory-vocal training which is typically started later in childhood or adolescence compared to instrumental music training. Singing has also received a lot of attention recently, since forms of singing have been shown to have positive effects on various neurological disorders (Wan et al., 2010b).

Regions in the superior temporal lobe, inferior frontal areas, and the associated premotor and motor regions are involved in the feedforward and feedback control of singing (Pantev et al., 1998; Maess et al., 2001; Levitin and Menon, 2003; Brown et al., 2004; Ozdemir et al., 2006). This fronto-temporal network of brain regions is connected via the arcuate fasciculus (AF), a prominent white-matter tract which, in its horizontal part, may share some components with the superior longitudinal fasciculus (SLF). The AF has direct fibers connecting the middle and superior temporal gyrus (STG) with inferior frontal regions, but may also have an indirect fiber system connecting the temporal lobe with the inferior parietal lobulus and then the parietal lobulus with frontal lobe regions (Catani et al., 2005; Glasser and Rilling, 2008). It should be noted that these putative "tracts" in fact represent tractography-derived pathways, and thus their fidelity to the anatomy is continuously contested, in particular with regards to whether or not the $\mathrm{AF}$ consists of direct fronto-temporal fibers or indirect fibers with synapses and relay stations in the parietal lobule (Catani et al., 2005; Frey et al., 2008; Glasser and Rilling, 2008). The AF and SLF have already been implicated in normal and abnormal vocal-motor activities. Fiber volume in the AF, and regional fractional anisotropy (FA - a measure of the degree of directional preference of water diffusion (Basser, 1995) values along its midpoint, are both lower in tone-deaf individuals (Loui et al., 2009). The phenotypical characteristic of 
tone-deaf individuals is that they cannot sing in tune and are not aware of their vocal feedback. The impairment of the AF in tonedeaf subjects supports it is presumed role in the feedforward and feedback control of vocal output.

In learning to associate motor actions with sounds and in training an auditory-motor feedback loop connecting the temporal with the frontal lobe, white-matter fiber bundles between these regions might change in size, volume, and composition. One common diffusion tensor imaging (DTI)-derived measure, FA, is a normalized measure expressing the directional diffusion of water protons; the more aligned fibers are within a tract, the higher the tract or regional FA value. FA changes within-group and FA differences between group can be regarded as a surrogate marker of structural adaptation in the white matter (Lindenberg et al., 2010). Adaptations of the AF have been described as a function of normal development (BarneaGoraly et al., 2005; Ashtari et al., 2007), and developmental delay has been found to be associated with disordered morphology of the AF, possibly reflecting delayed myelination (Sundaram et al., 2008).

Previous research has identified regions that may control and be strengthened by vocal training (Zarate and Zatorre, 2008; Zarate et al., 2010). In addition to the STG and its reciprocal connections with the inferior premotor and posterior IFG, the middle temporal gyrus (MTG) also has prominent connections with the inferior frontal gyrus (IFG). These two branches can be thought of as two separate branches with possibly slightly different functions in the auditory-motor mapping and auditory-motor control system.

Since the connectivity between STG, MTG, and IFG allows for the planning of complex motor sequences as well as the monitoring and correction of feedback and feedforward motor commands (Guenther et al., 2006), we hypothesized that the connectivity between these regions will be enhanced in musicians as a result of training-dependent or use-dependent plasticity in auditory-motor integration, and perhaps particularly in singers, given their specific auditory-vocal training requiring intense and precise auditorymotor feedforward and feedback control. We examined the connectivity of the AF and its dorsal and ventral branch, which connects the STG, MTG, and IFG, using DTI, an MR imaging technique that enables the visualization and quantitative assessment of whitematter pathways in the brain (Basser et al., 1994, 2000; Makris et al., 1997, 2005; Catani and Thiebaut De Schotten, 2008). Since finegrained perception and feedback control of pitch and motor actions are both important aspects of musical training, we hypothesized that singers and instrumental musicians would exhibit structural adaptations in the AF relative to non-musicians, although the AF and its subdivisions in both hemispheres might be differentially affected considering the fine-grained sensory-motor mapping of sounds to articulation which we assumed is an important role for the dorsal branch of the AF.

\section{MATERIALS AND METHODS \\ PARTICIPANTS}

Twenty-two musicians were recruited through posting ads on Craigslist, bulletins at local music conservatories, and choral groups in the greater Boston area. Eleven of these individuals were either professional singers or reported to be in training to be professional singers; these singers did have some experience in playing musical instruments, such as piano $(n=5)$, cello (2), trombone (1), trumpet
(1), flute (1), and clarinet (1) but were not actively practicing these musical instruments at the time of this study or in the year prior to enrollment. This group of singers was contrasted with a group of 11 instrumental musicians who did not report singing as a primary musical activity, did not have any formal singing training and were not part of any singing group, but had an equal amount of musical experience as determined by their number of years of musical training. This group's instruments included piano $(n=4)$, violin (4), cello (1), and trombone (2). These two groups of 11 musicians each were contrasted with a third group of 11 participants without any significant instrumental music training (defined as less than 1 year of instrumental music training throughout their life, but not in the year before enrollment in this study), any formal singing training, and no participation in any singing groups. This group of non-musicians was recruited through the same mechanisms as the other groups and was matched to the musician groups with regards to age, gender, and IQ. Singers, instrumental musicians, and non-musicians were similar in the mean age [ singers $=25.3$ $(\mathrm{SD}=2.7)$ years; instrumental musicians $=27.7(\mathrm{SD} 7.6)$ years; non-musicians $=27.5$ (SD 10.3) years], and gender $(5 \mathrm{~m} / 6 \mathrm{f}$ for instrumentalists, $3 \mathrm{~m} / 8 \mathrm{f}$ for singers, and $5 \mathrm{~m} / 6 \mathrm{f}$ for non-musicians). Singers and instrumentalists were matched for number of years in musical training (singers' mean $=15, \mathrm{SD}=5$; instrumentalists' mean $=14.3, \mathrm{SD}=9.09$ ) and age of training onset (singers' mean $=6.6, \mathrm{SD}=2.4$; instrumentalists' mean $=7.4, \mathrm{SD}=4.4$ ). The three groups were also matched with regards to their IQ as assessed by Shipley's verbal and abstract scaled composite score (Shipley, 1940) (singers' mean $=111, \mathrm{SD}=14$, instrumentalists' mean $=114, \mathrm{SD}=10$, non-musicians' mean $=111, \mathrm{SD}=13$ ). This study was approved by the Institutional Review Board of the Beth Israel Deaconess Medical Center and all participants provided written informed consent.

\section{IMAGE ACQUISITION}

MR images were obtained using a 3-Tesla General Electric scanner. MR sequences included a strongly T1-weighted data set with a voxel resolution of $0.93 \mathrm{~mm} \times 0.93 \mathrm{~mm} \times 1.5 \mathrm{~mm}$. DTI was performed using a diffusion-weighted, single-shot, spin-echo, echo-planar imaging sequence $(\mathrm{TE} 1=86.9 \mathrm{~ms}$, relaxation time $=10,000 \mathrm{~ms}$, field of view $=240 \mathrm{~mm}$, matrix size $=94 \times 94$ voxels, no skip, $\mathrm{NEX}=1$, axial acquisition, voxel dimensions $=2.5 \mathrm{~mm} \times 2.5 \mathrm{~mm} \times 2.6 \mathrm{~mm}$ ). Thirty non-collinear directions with a $b$ value of $1000 \mathrm{~s} / \mathrm{mm}^{2}$ and six volumes with a $b$ value of $0 \mathrm{~s} / \mathrm{mm}^{2}$ were acquired.

\section{DATA PROCESSING}

FMRIB's FSL suite was used for pre-processing of raw images (Smith et al., 2004). A 3D affine registration was applied to correct for eddy currents and head motion (Jenkinson and Smith, 2001) followed by BET for brain extraction (Smith, 2002). Eigenvectors and eigenvalues of diffusion tensors were estimated at each voxel and FA was calculated using the dtifit function in FSL. Axial diffusivity, a parameter reflecting the principal direction of diffusion in white matter (Song et al., 2002), was estimated using the first eigenvalue of the diffusion tensor, $\lambda_{1}$. The second and third eigenvalues were averaged and referred to as the radial diffusivity $\left(\lambda_{2}+\lambda_{3}\right) / 2$ (Basser, 1995; Xue et al., 1999; Basser et al., 2000; Song et al., 2002). Following this, a probability distribution for fiber direction was 
calculated for each brain voxel using the bedpostx function in FSL (Behrens et al., 2003). To allow for fiber crossings, estimates of two directions per voxel were allowed (Behrens et al., 2007).

\section{TRACTOGRAPHY - ARCUATE FASCICULUS}

Regions of interest (ROIs) were drawn by hand, on a single sagittal slice of the native FA image of each subject, according to major anatomical landmarks, shown in Figure 1. For each hemisphere, ROIs were drawn on white matter underlying the gray matter in three regions: posterior superior temporal gyrus (pSTG), posterior middle temporal gyrus (pMTG), and posterior inferior frontal gyrus (pIFG). Two separate one-way ANOVAs (with the dependent measure of ROI volume for one, and FA for the other) showed no significant effect of group on either: ROI mean volumes for singers: $124.2 \mathrm{~mm}^{3}(\mathrm{SD}=41.7)$; instrumentalists' $=136 \mathrm{~mm}^{3}(\mathrm{SD}=39)$; non-musicians' mean $=124.1 \mathrm{~mm}^{3}(\mathrm{SD}=41.7)$. FA values: singers' mean $=0.411, \mathrm{SD}=0.067$, instrumentalists' mean $=0.422$, $\mathrm{SD}=0.059$, non-musicians' mean $=0.401, \mathrm{SD}=0.062$ ).

Probabilistic tractography was applied to constrain white-matter tracts to the dorsal and ventral branches of the AF in each hemisphere. Tractography was initiated from one seed region to one-way point mask region using the probtrackx function in FSL in two separate steps: (1) Tracts traced from the seed region of STG to the waypoint mask of IFG were identified as the dorsal AF. (2) Tracts traced from the seed region of MTG to the waypoint mask of IFG were identified as the ventral AF. These resulting tracts were further masked by setting an intensity threshold of the median intensity values of each tract, and eliminating voxels with intensity values below that threshold. While this provides a way to threshold the tracts similarly across subjects, choosing a cut-off intensity value is somewhat arbitrary, and since there is currently no convention for setting this value (Giorgio et al., 2010), we chose the median intensity value for each tract. To compute tract volume, we used the number of voxels in each tract after applying the cut-off intensity value and multiplied the number of voxels by the voxel size (Loui et al., 2011a,b).

\section{STATISTICAL COMPARISONS - ARCUATE FASCICULUS}

For each tract (dorsal AF, ventral AF) in each hemisphere, measures of volume and FA were extracted and compared using a threeway ANOVA with the between-subjects factor of GROUP (with three levels: singers, instrumentalists, and non-musicians) and the within-subject factors of HEMISPHERE (left vs. right) and TRACT (dorsal vs. ventral).
As a control for results from tractography of the AF, we extracted mean FA and volume for non-zero voxels from each subjects' wholebrain FA image, and compared the three groups' means to control for possible whole-brain differences between groups using two one-way ANOVAs (one for FA and one for volume).

\section{RESULTS}

\section{TRACT STATISTICS - ARCUATE FASCICULUS}

Tract volume was largest in singers, especially in the left hemisphere. This was confirmed using a three-way ANOVA on the dependent variable of tract volume with the between-subjects factor of GROUP (with three levels: singers, instrumentalists, and non-musicians) and the within-subject factors of HEMISPHERE (left vs. right) and TRACT (dorsal vs. ventral). This ANOVA revealed an overall significant effect of GROUP $[F(2,120)=9.8$, $p<0.001$ ], confirming that tract volume was highest in singers, second-highest in instrumentalists, and lowest in non-musicians. Furthermore, the same ANOVA revealed a significant main effect of HEMISPHERE $[F(1,120)=9.2, p=0.003]$, with tract volume being higher in the left than in the right hemisphere. Finally, the interaction between GROUP and HEMISPHERE was significant $[F(2,120)=4.4, p=0.014]$, confirming that the tract volume in the left hemisphere was especially higher among singers relative to the other two groups. These results are summarized in Figure 2.

In order to ascertain that tract volume in the left hemisphere was especially higher among singers relative to the other two groups, we performed pairwise post hoc comparison for each tract between singers and instrumentalists, singers and non-musicians, and between instrumentalists and non-musicians applying appropriate Bonferroni corrections. The dorsal branch of the left AF showed a significantly higher volume in singers relative to instrumentalists $[t(10)=3.36, p=0.007]$, surviving Bonferroni corrections for two hemispheres and two branches of the AF in each hemisphere. The same branch's volume is also significantly higher in singers relative to non-musicians $[t(10)=3.76, p=0.004]$, also surviving post hoc corrections for the four branches of the AF.

Tract FA was lower in singers than in instrumentalists, especially in the dorsal branch of the left hemisphere. This was tested using a three-way ANOVA on the dependent variable of FA with the between-subjects factor of GROUP and the within-subjects factors of HEMISPHERE and TRACT. The main effect of GROUP was significant $[F(2,120)=8.174, p<0.001]$, confirming that FA was highest in instrumentalists, followed by singers and then by nonmusicians, although this finding did not survive strict correction

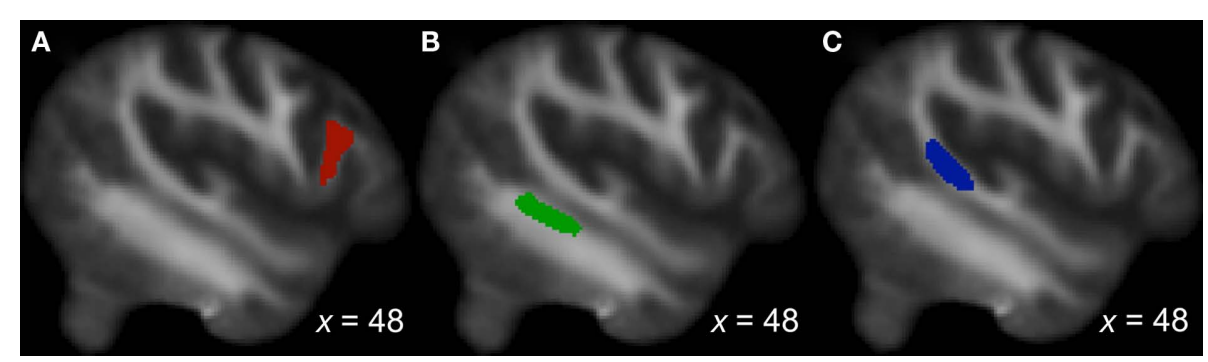

FIGURE 1 | Region of interest locations displayed on the FA images: (A) IFG, (B) MTG, (C) STG. 
for multiple tests. The main effect of TRACT was significant $[F(1,120)=12.49, p=0.001]$, confirming that tract FA was lower in the dorsal branch relative to the ventral branch. Finally, an interaction between GROUP and HEMISPHERE showed a trend toward significance $[F(2,120)=2.73, p=0.069]$, showing that FA was lower in the left hemisphere of singers compared to both hemispheres of instrumentalists. These results are summarized in Figure 3.

To explore the potentially differing contributions of axial and radial diffusivities to the observed FA difference between singers and instrumentalists, we extracted the singers' and instrumentalists' left dorsal AF's mean axial $\left(\lambda_{1}\right)$ and radial $\left[\left(\lambda_{2}+\lambda_{3}\right) / 2\right]$ diffusivities. A direct comparison of these parameters between singers and instrumentalists suggested that the finding of lower FA was strongly due to higher radial diffusivity $\left[\left(\lambda_{2}+\lambda_{3}\right) / 2\right]$ in singers relative to instrumentalists $[t(20)=2.7, p=0.01]$, and not due to differences in axial diffusivity $[t(20)=1.01, p=0.33]$.

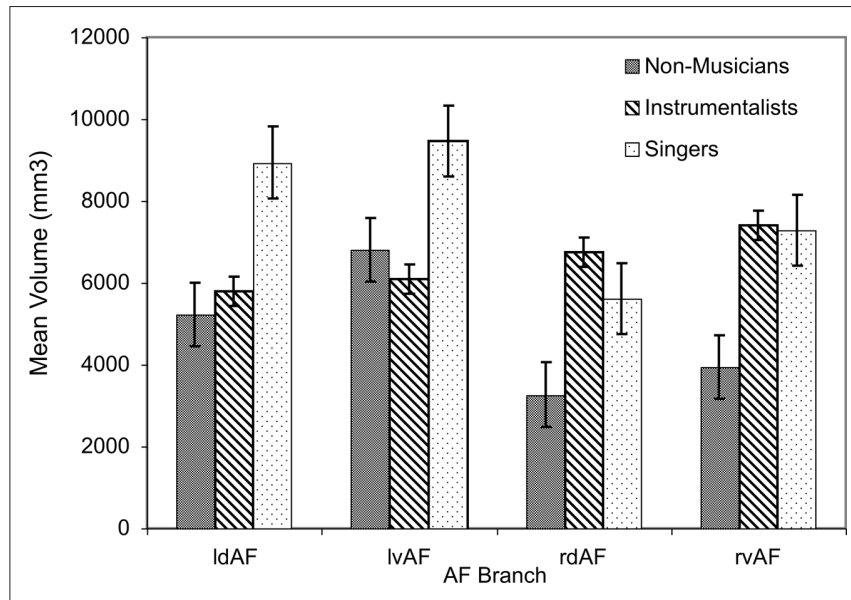

FIGURE 2 | Mean volume for all branches of the AF in both hemispheres for all groups ( $\mathbf{l}=$ left, $\mathbf{r}=$ right, $\mathbf{d}=\mathbf{\text { dorsal}}, \mathbf{v}=\mathbf{\text { ventral}}$ ). Error bars represent SE of the mean.

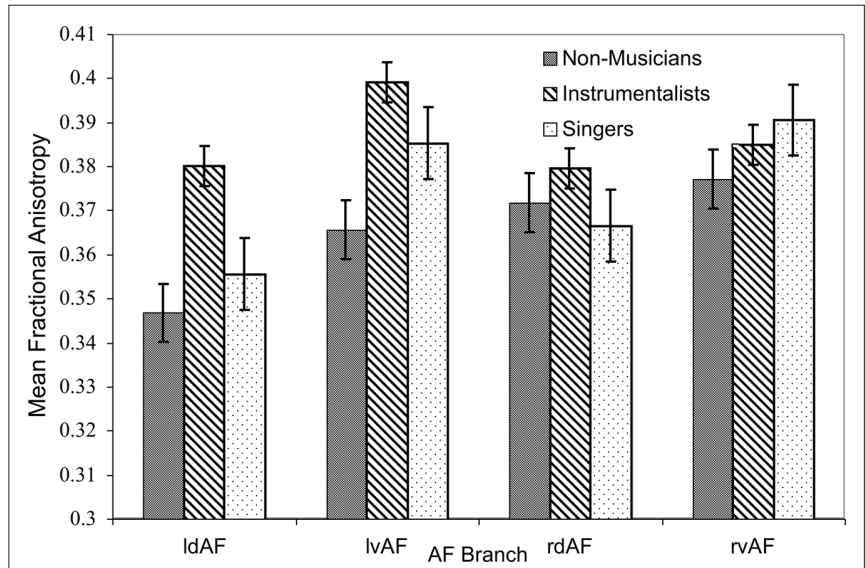

FIGURE 3 | Mean FA for all branches of the AF in both hemispheres for all groups ( $=$ left, $\mathbf{r}=$ right, $\mathbf{d}=\mathbf{d}$ dorsal, $\mathbf{v}=$ ventral). Error bars represent $\mathrm{SE}$ of the mean.
To test whether the between-group differences in the AF could be explained by whole-brain differences in FA or volume, separate oneway ANOVAs were conducted on the dependent variables of FA and volume. Results showed no significant between-group differences in whole-brain FA or volume (all ps > 0.1), suggesting that differences in the AF cannot be explained by whole-brain differences.

\section{FA ALONG THE ARCUATE FASCICULUS}

Having identified the left dorsal AF as the main tract of interest that showed differences between singers and matched instrumental musician controls, we further sought to identify the region along the AF that shows maximal between-group differences. Visual comparison of the FA maps of tracts identified in singers and instrumentalists (Figure 4) suggested that a part of the longitudinal portion of the left dorsal AF showed the strongest FA differences between groups.

To explore this observed difference along the tract, the longitudinal portion of the left dorsal AF was divided into 4 bins of 10 coronal slices each and the mean FA from each bin (mean FA of 10 coronal slices) was extracted for each subject, and compared between singers and instrumentalists using a two-tailed $t$-test. The comparison revealed significantly lower FA for the singers in bin $3[y=-22 \mathrm{~mm}$ to $y=-13 \mathrm{~mm}, t(9)=4.29, p=0.002$ ], around the midpoint of the longitudinal portion of the left dorsal AF. Figure 5 shows bin-bybin comparisons between singer and instrumental groups in the longitudinal portion of the left dorsal AF, whereas Figures 6 and 7 show the entire left dorsal AF tract in axial (Figure 6) and sagittal (Figure 7) views, highlighting the section that is significantly lower in FA in singers than controls: on average, singers possessed lower FA in the longitudinal portion of the left dorsal AF, and more specifically in bin 3 (from $y=-22 \mathrm{~mm}$ to $y=-13 \mathrm{~mm}$ ).

\section{BEHAVIORAL CORRELATES OF FA DIFFERENCES}

To explore the relationship between singers and FA values in the left dorsal AF, we tested the hypothesis that the number of years of singing training is predictive of FA value for the left dorsal AF using a linear regression (shown in Figure 8). Within the singer group only, the reported number of years of singing training inversely predicted the FA value of each individual's left dorsal AF $\left(R^{2}=-0.387\right.$, $p=0.04$ ). For both FA and volume, a similar relationship was not observed in the left dorsal AF of control musicians $\left(R^{2}=-0.08\right.$, $p>0.1)$, nor for any other tracts in both singers' and instrumentalists (all $\left|R^{2}\right| \mathrm{s}<0.16$, all $p s>0.2$ ).

\section{DISCUSSION}

We report a DTI comparison between singers, instrumental musicians, and non-musicians. Our results showed that the AF, a bundle of white-matter fibers that connects regions of the brain known to be involved in sound perception and production as well as the feedforward and feedback control and vocal output, has a larger volume and higher FA values in musicians (both singers and instrumentalists) relative to non-musicians. Among musicians, singers showed significantly higher volume in the dorsal and ventral branches of the left AF, and lower FA in the left dorsal AF, relative to instrumentalists. In contrast, GROUP had no effect on whole-brain FA measures between the three groups, suggesting that effects could not be explained by whole-brain differences. A bin-by-bin analysis 


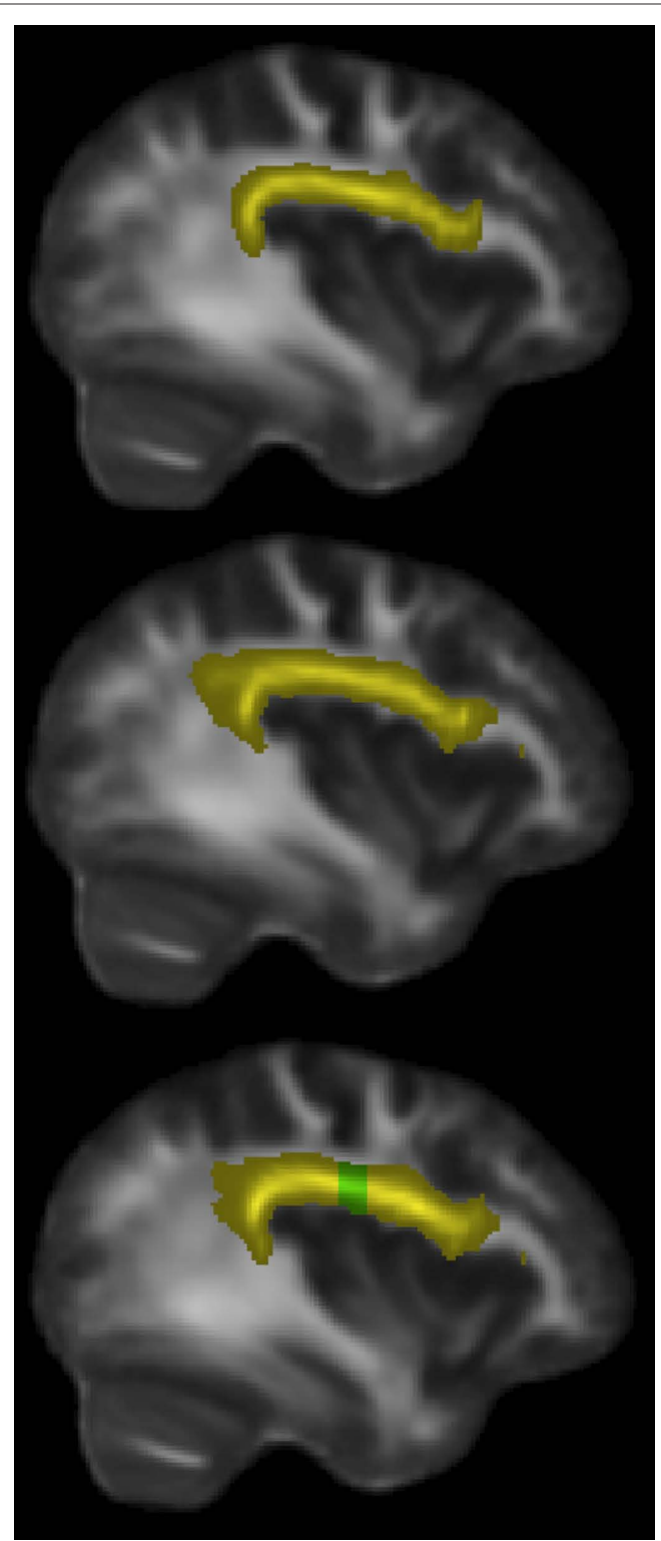

FIGURE 4 | Sagittal slice $x=38 \mathrm{~mm}$, showing left dorsal AF in yellow: instrumental controls (top). Singers (middle). All musicians (bottom), with region showing significant FA differences at $y=-22$ to $-13 \mathrm{~mm}$, shown in green.

of FA differences in the longitudinal portion of the AF among musicians shows significantly lower FA among singers along the midpoint of the left dorsal AF, relative to instrumentalists.

The finding of higher FA in musicians compared to non-musicians, but lower FA in singers relative to instrumental musicians provides support for auditory-motor training-induced differences in the AF for musicians in general and singers in particular. The lower FA may reflect less aligned fibers or more fiber crossings among singers along the course of the AF. While DTI does not resolve the directionality of fiber tracts, tractography methods make it possible to infer tract volume based on identified tracts that connect several endpoints of gray matter (Giorgio et al., 2010).

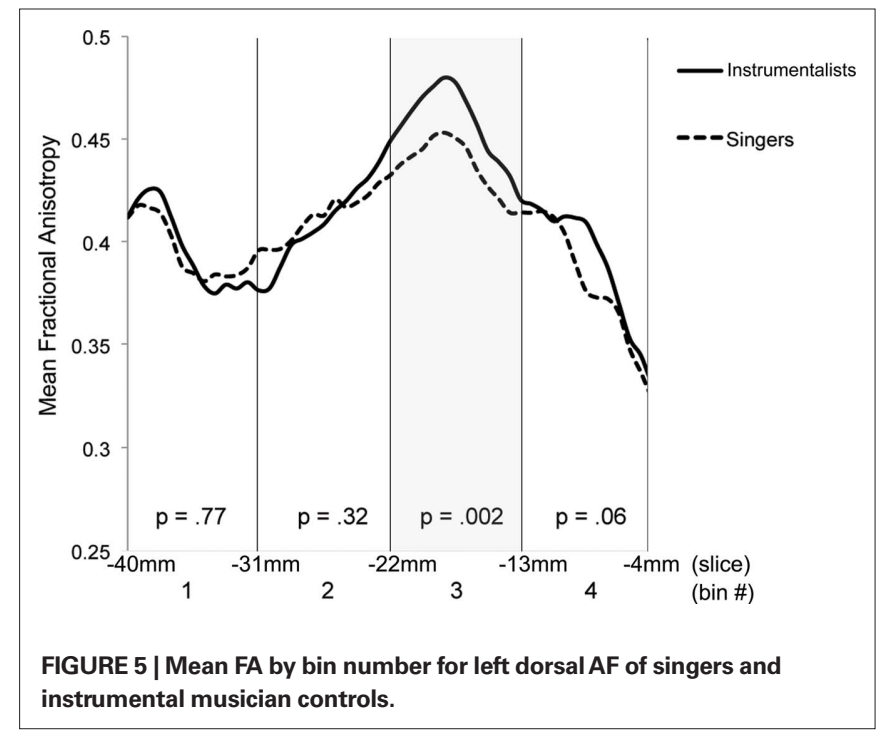

By using seed regions in the superior and middle temporal gyri, and waypoint masks in the IFG of each hemisphere in each brain, we were able to identify the dorsal and ventral branches of the left and right AF. Both dorsal and ventral branches of the left AF were identified as being larger in singers than in instrumentalists, with stronger effects in the dorsal branch. Interestingly, this effect was only observed in the left hemisphere.

Previous research has shown that individuals with difficulties in pitch perception and production, i.e., tone-deaf individuals, have diminished white-matter volume in their right AF, with pitch perception abilities being correlated with the dorsal branch of the right AF and pitch perception-production mismatch being correlated with the right ventral branch (Loui et al., 2009), suggesting that fine-grained control of pitch perception and production abilities are related to the dorsal branch (i.e., STG $\leftrightarrow$ IFG connections). In contrast, both singers and instrumental musicians in this study have expertise in pitch perception and production, as the instrumental group is matched for number of years of musical training. It is only when both the singers and instrumentalists are compared with nonmusicians that differences in the right AF are observed. This might suggest that the right AF shows more of a domain general adaptation effect in activities that involve matching sounds with actions independent on whether these actions are articulatory actions or hand actions. The left AF and in particular the dorsal branch of the left AF showed the most profound differences comparing singers with instrumentalists. Although this finding is somewhat surprising, it suggests that the left AF, which already shows an adaptation when individuals acquire language (Barnea-Goraly et al., 2005; Ashtari et al., 2007) and is usually larger and more complex than the right AF (Vernooij et al., 2007; Glasser and Rilling, 2008), might be the structure that adapts the most to the specific requirements of vocal-motor and auditory-motor integration.

Furthermore, it is also possible that the dorsal (STG $\leftrightarrow$ IFG) and ventral (MTG $\leftrightarrow$ IFG) branches have different functions and therefore show different adaptations. The ventral (MTG-IFG) branch could be a fast and coarse system that does not rely on precise auditory feedback while the dorsal (STG $\leftrightarrow$ IFG) branch could be 


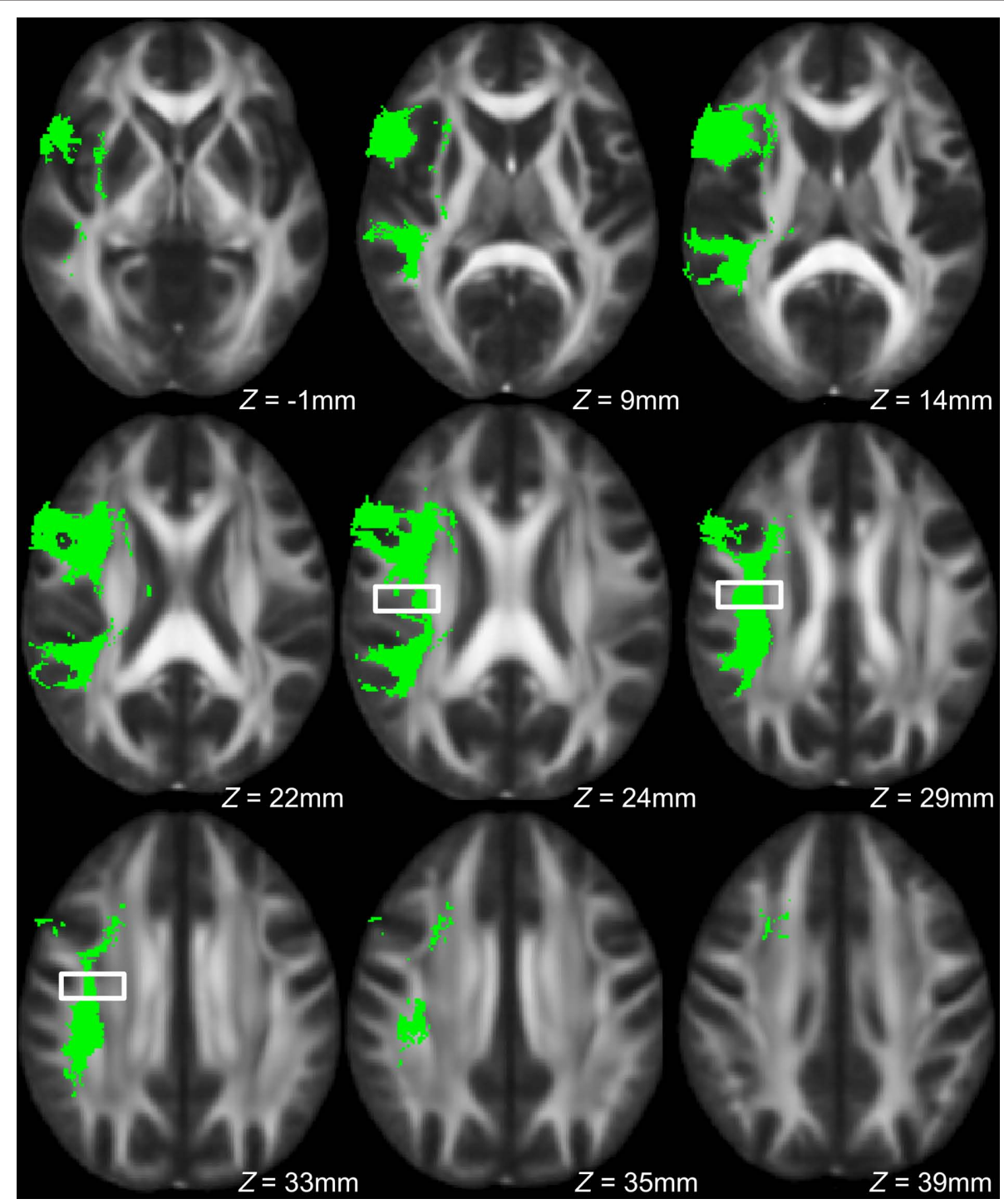

FIGURE 6 | Axial view of left dorsal AF across all subjects in green, with region showing significant between-group FA differences indicated by the box.

a slower, more precise system that is under conscious control and heavily relies on auditory feedback to make adjustments in the auditory-motor mapping of vocal output.

Similar findings of lower FA values in white-matter regions have been reported in other studies comparing experts in a particular domain with non-experts. For example, simultaneous interpreters (individuals who translate a source's speech in real-time) were reported to have lower FA relative to controls, in subregions of their corpus callosum, as well as in the white matter underlying the left anterior insula and inferior parietal lobe, regions thought to be involved in articulatory control and sensory-motor mapping for speech (Elmer et al., 2010).

Diffusion tensor imaging provides in vivo measures of whitematter connectivity through its parameters of FA, axial diffusivity, radial diffusivity, and shape and volume of white-matter tracts. FA is a scalar measure that characterizes the degree of directional preference in diffusion of water, and is affected by the degree of myelination and axonal coherence. Axial diffusivity is said to be related to the integrity of the axons, whereas radial diffusivity is said to be related to the degree of myelination (Song et al., 2002; Schaechter et al., 2009). However there is much debate about the validity of these associations especially when applied to graywhite-matter boundaries or regions with multiple crossing fibers (Wheeler-Kingshott and Cercignani, 2009).

The finding of lower FA due to higher radial diffusivity in singers, in particular in bin 3 of the tract (around the midpoint of the longitudinal portion of the $\mathrm{AF}$, most proximal to regions of the motor and somatosensory cortices) suggests that the singers' AFs may be adapted to allow for more connectivity between the primary nodes of the AF (STG/MTG and IFG), the motor/somatosensory system, and/or the inferior parietal lobule via the AF's putative "indirect pathway" (as described in Catani et al., 2005). Since the FA difference is most strong in bin 3 and bin 3 roughly coincides with the location of the pre- and postcentral gyrus, this suggests 


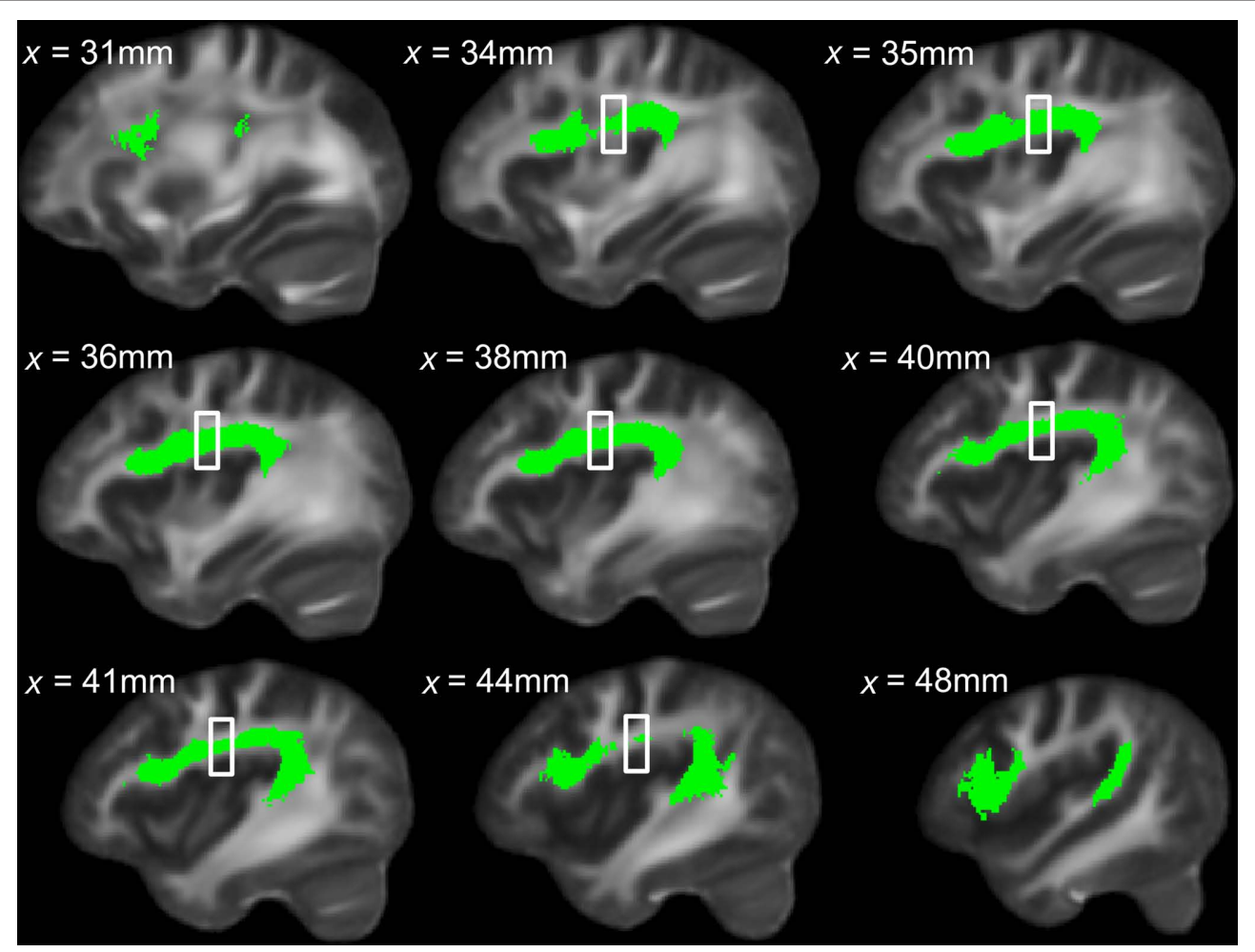

FIGURE 7 | Sagittal view of left dorsal AF across all subjects in green, with region showing significant between-group FA differences indicated by the box.

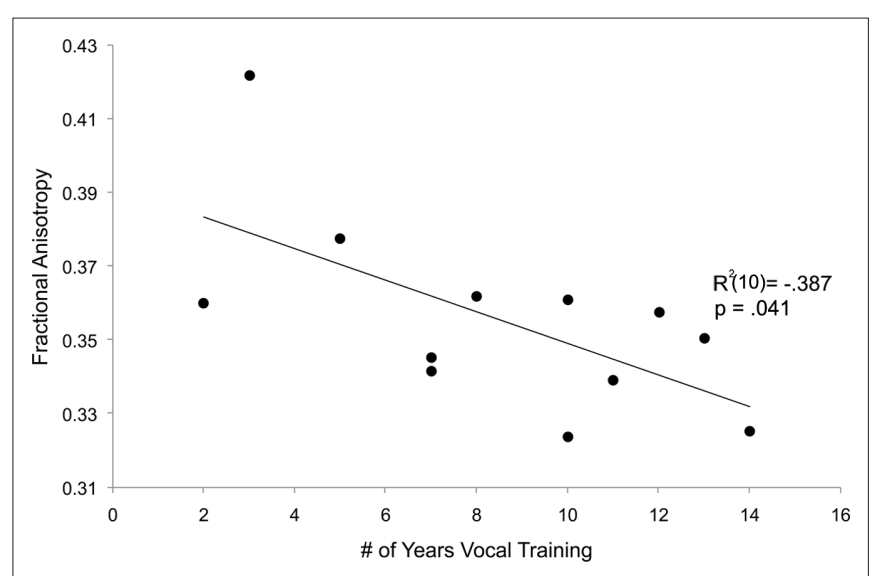

FIGURE 8 | Numbers of years of vocal training (singers' group only) inversely predicts mean FA value of the left dorsal AF.

that at least part of the increase in radial diffusivity in singers may be reflective of less alignment in one direction and possibly more branching in other directions (e.g., perpendicular to the axial plane of the tract, toward the pre- and postcentral gyrus). Differences in white-matter microstructure of this kind (increased radial diffusivity with no difference in axial diffusivity) are most frequently interpreted as indicating differences in myelination (Song et al., 2002; Alexander et al., 2007; Jito et al., 2008), but changes in myelin or axon density due to axonal sprouting are also possible (Dancause et al., 2005; Carmichael, 2006), although the extent to which DTI is sensitive to such phenomena is not known. Nevertheless, enhanced connectivity of this kind in singers might be an adaptation for increased sensitivity for feedback information from the inferior parts of the somatosensory cortex (homuncular representations of sensory feedback from speech areas, e.g., proprioception of jaws, tongue, and lips), as well as feedforward information provided to the inferior regions of the inferior motor/premotor strip (homuncular representations of the tongue, jaw, lips, and larynx). This interaction of feedback and feedforward information in which the AF may play a critical role has been described in detail in Guenther et al. (2006) for syllable production in speaking and might be similar for intoned syllable production in singing. Functional neuroimaging studies have shown an extended activation after vocal skills training involving inferior motor/premotor and somatosensory regions (Kleber et al., 2010). In contrast to instrumental musicians, who exercise fine non-vocal-motor control while engaging their vocal system minimally during a performance, singers must always monitor their breathing as well as proprioception from their vocal apparatus. This added cognitive demand necessitates stronger connectivity between temporal, inferior frontal, as well as inferior motor/premotor, and inferior somatosensory regions; this may be reflected in differing white-matter architecture in the AF of singers, relative to instrumentalists.

Our results suggest that musical training, particularly vocal training, is associated with structural adaptations in the AF: a tract that is important for linguistic as well as musical functions. In individuals who receive primarily vocal musical training, adaptations are observed especially in the left dorsal AF, which is larger in volume but lower in FA among singers (relative to instrumentalists). This combination of DTI differences may reflect more fiber 
crossings in white-matter regions between STG and IFG, possibly reflecting a more complex pattern of connectivity for the $\mathrm{AF}$ that may be associated with vocal training as compared to other kinds of musical training.

The present study provides further support for the use of musically trained individuals, especially singers, as a model for structural as well as functional adaptations of the auditory-motor system (Schlaug, 2001) by showing structural differences between the brains of those engaged in specific types of musical training (vocal vs. instrumental). The intensive training that is typical in learning to sing may be translated into neurorehabilitation programs for aphasic patients and other groups of impaired vocal output (Schlaug et al., 2008; Wan and Schlaug, 2010a; Wan et al., 2010a), whose whitematter pathways in the undamaged hemisphere show adaptations in

\section{REFERENCES}

Alexander, A. L., Lee, J. E., Lazar, M., and Field, A. S. (2007). Diffusion tensor imaging of the brain. Neurotherapeutics 4, 316-329.

Ashtari, M., Cervellione, K. L., Hasan, K. M., Wu, J., Mcilree, C., Kester, H., Ardekani, B. A., Roofeh, D., Szeszko, P. R., and Kumra, S. (2007). White matter development during late adolescence in healthy males: a cross-sectional diffusion tensor imaging study. Neuroimage 35, 501-510.

Barnea-Goraly, N., Menon, V., Eckert, M., Tamm, L., Bammer, R., Karchemskiy, A., Dant, C. C., and Reiss, A. L. (2005). White matter development during childhood and adolescence: a crosssectional diffusion tensor imaging study. Cereb. Cortex 15, 1848-1854.

Basser, P. J. (1995). Inferring microstructural features and the physiological state of tissues from diffusion-weighted images. NMR Biomed. 8, 333-344.

Basser, P. J., Mattiello, J., and Lebihan, D. (1994). MR diffusion tensor spectroscopy and imaging. Biophys. J. 66, 259-267.

Basser, P. J., Pajevic, S., Pierpaoli, C., Duda, J., and Aldroubi, A. (2000). In vivo fiber tractography using DT-MRI data. Magn. Reson. Med. 44, 625-632.

Behrens, T. E., Berg, H. J., Jbabdi, S., Rushworth, M. F., and Woolrich, M. W. (2007). Probabilistic diffusion tractography with multiple fibre orientations: what can we gain? Neuroimage 34, 144-155.

Behrens, T.E., Woolrich, M.W., Jenkinson, M., Johansen-Berg, H., Nunes, R. G., Clare, S., Matthews, P. M., Brady, J. M., and Smith, S. M. (2003). Characterization and propagation of uncertainty in diffusion-weighted MR imaging. Magn. Reson. Med. 50, 1077-1088.

Bengtsson, S. L., Nagy, Z., Skare, S., Forsman, L., Forssberg, H., and Ullen, F. (2005). Extensive piano practicing has regionally specific effects on white matter development. Nat. Neurosci. 8, 1148-1150.

Brown, S., Martinez, M. J., Hodges, D. A., Fox, P. T., and Parsons, L. M. (2004). The song system of the human brain. Brain Res. Cogn. Brain Res. 20, 363-375.

Carmichael, S. T. (2006). Cellular and repair after stroke: making waves. Ann. Neurol. 59, 735-742.

Catani, M., Jones, D. K., and Ffytche, D. H. (2005). Perisylvian language networks of the human brain. Ann. Neurol. 57, 8-16.

Catani, M., and Thiebaut De Schotten, M. (2008). A diffusion tensor imaging tractography atlas for virtual in vivo dissections. Cortex 44, 1105-1132.

Dancause, N., Barbay, S., Frost, S. B., Plautz, E. J., Chen, D., Zoubina, E. V., Stowe, A. M., and Nudo, R. J. (2005). Extensive cortical rewiring after brain injury. J. Neurosci. 25, 10167-10179.

Elmer, S., Hanggi, J., Meyer, M., and Jancke, L. (2010). Differential language expertise related to white matter architecture in regions subserving sensory-motor coupling, articulation, and interhemispheric transfer. Hum Brain Mapp. PMID: 21162044. [Epub ahead of print].

Frey, S., Campbell, J. S., Pike, G. B., and Petrides, M. (2008). Dissociating the human language pathways with high angular resolution diffusion fiber tractography. J. Neurosci. 28, 11435-11444.

Giorgio, A., Santelli, L., Tomassini, V., Bosnell, R., Smith, S., De Stefano, N., and Johansen-Berg, H. (2010). Agerelated changes in grey and white matter structure throughout adulthood. Neuroimage 51, 943-951.

Glasser, M. F., and Rilling, J. K. (2008). DTI tractography of the human brain's language pathways. Cereb. Cortex 18, 2471-2482.

Guenther, F. H., Ghosh, S. S., and Tourville, J. A. (2006). Neural modmolecular mechanisms of neural

response to intense intonation-based speech therapy (Schlaug et al., 2009b; Wan et al., 2010b). By characterizing the differences in whitematter connectivity between singers and non-singer controls who are matched in musical training, the present study suggests that intense musical training, particularly vocal-motor training, has an effect on the macro- and microstructural anatomy of vocal-motor networks.

\section{ACKNOWLEDGMENTS}

The study was supported by research grants from NIH/NIDCD (RO1 DC009823 and RO1 DC008796). Gus Halwani acknowledges support from a training grant (T32 DC00038) from NIH/ NIDCD. Furthermore, support from the Nancy Lurie Marks Family Foundation and from the family of Rosalyn and Richard Slifka is also acknowledged.

eling and imaging of the cortical interaction underlying syllable production. Brain Lang. 96, 280-301.

Hyde, K. L., Lerch, J., Norton, A., Forgeard, M., Winner, E., Evans, A. C., and Schlaug, G. (2009). Music training shapes structural brain development. J. Neurosci. 29, 3019-3025.

Jenkinson, M., and Smith, S. (2001). A global optimisation method for robust affine registration of brain images. Med. Image Anal. 5, 143-156.

Jito, J., Nakasu, S., Ito, R., Fukami, T. Morikawa, S., and Inubushi, T. (2008). Maturational changes in diffusion anisotropy in the rat corpus callosum: comparison with quantitative histological evaluation. J. Magn. Reson. Imaging 28, 847-854.

Kleber, B., Veit, R., Birbaumer, N., Gruzelier,J., and Lotze, M. (2010). The brain of opera singers: experiencedependent changes in functional activation. Cereb. Cortex 20, 1144-1152.

Koelsch, S., Gunter, T. C., Wittfoth, M., and Sammler, D. (2005). Interaction between syntax processing in language and in music: an ERP Study. J. Cogn. Neurosci. 17, 1565-1577.

Levitin, D. J., and Menon, V. (2003). Musical structure is processed in "language" areas of the brain: a possible role for Brodmann Area 47 in temporal coherence. Neuroimage 20, 2142-2152.

Lindenberg, R., Renga, V., Zhu, L. L., Betzler, F., Alsop, D., and Schlaug, G. (2010). Structural integrity of corticospinal motor fibers predicts motor impairment in chronic stroke. Neurology 74, 280-287.

Loui, P., Alsop, D., and Schlaug, G. (2009). Tone deafness: a new disconnection syndrome? J. Neurosci. 29, 10215-10220.

Loui, P., Li, H. C., Hohmann, A., and Schlaug, G. (2011a). Enhanced cortical connectivity in absolute pitch musicians: a model for local hyperconnectivity. J. Cogn. Neurosci. 23, 1015-1026.
Loui, P., Li, H.C., and Schlaug, G. (2011b). White matter integrity in right hemisphere predicts pitch related grammar learning. Neuroimage 55, 500-507.

Maess, B., Koelsch, S., Gunter, T. C., and Friederici, A. D. (2001). Musical syntax is processed in Broca's area: an MEG study. Nat. Neurosci. 4, 540-545.

Makris, N., Kennedy, D. N., Mcinerney, S., Sorensen, A. G., Wang, R., Caviness, V. S. Jr., and Pandya, D. N. (2005). Segmentation of subcomponents within the superior longitudinal fascicle in humans: a quantitative, in vivo, DT-MRI study. Cereb. Cortex 15, 854-869.

Makris, N., Worth, A. J., Sorensen, A. G., Papadimitriou, G. M., Wu, O., Reese, T. G., Wedeen, V. J., Davis, T. L., Stakes, J.W., Caviness, V.S., Kaplan, E., Rosen, B. R., Pandya, D. N., and Kennedy, D. N. (1997). Morphometry of in vivo human white matter association pathways with diffusion-weighted magnetic resonance imaging. Ann. Neurol. 42, 951-962.

Moreno, S., Marques, C., Santos, A., Santos, M., Castro, S. L., and Besson, M. (2009). Musical training influences linguistic abilities in 8-year-old children: more evidence for brain plasticity. Cereb. Cortex 19, 712-723.

Oechslin, M. S., Imfeld, A., Loenneker, T., Meyer, M., and Jancke, L. (2009). The plasticity of the superior longitudinal fasciculus as a function of musical expertise: a diffusion tensor imaging study. Front. Hum. Neurosci. 3:76. doi: 10.3389/neuro.09.076.2009

Ozdemir, E., Norton, A., and Schlaug, G. (2006). Shared and distinct neural correlates of singing and speaking. Neuroimage 33, 628-635.

Pantev, C., Oostenveld, R., Engelien, A., Ross, B., Roberts, L. E., and Hoke, M. (1998). Increased auditory cortical representation in musicians. Nature $392,811-814$. 
Ross, D. A., Olson, I. R., and Gore, J. C. (2003). Cortical plasticity in an early blind musician: an fMRl study. Magn. Reson. Imaging 21, 821-828.

Schaechter, J. D., Fricker, Z. P., Perdue, K. L., Helmer, K. G., Vangel, M. G., Greve, D. N., and Makris, N. (2009). Microstructural status of ipsilesional and contralesional corticospinal tract correlates with motor skill in chronic stroke patients. Hum. Brain Mapp. 30, 3461-3474.

Schlaug, G. (2001). The brain of musicians. A model for functional and structural adaptation. Ann. N. Y.Acad. Sci. 930, 281-299.

Schlaug, G., Forgeard, M., Zhu, L., Norton, A., and Winner, E. (2009a). Traininginduced neuroplasticity in young children. Ann. N. Y. Acad. Sci. 1169, 205-208.

Schlaug, G., Marchina, S., and Norton, A. (2009b). Evidence for plasticity in white-matter tracts of patients with chronic Broca's aphasia undergoing intense intonation-based speech therapy. Ann. N. Y.Acad. Sci. 1169, 385-394.

Schlaug, G., Marchina, S., and Norton, A. (2008). From singing to speaking: why singing may lead to recovery of expressive language function in patients with Broca's aphasia. Music Percept. 25, 315-323.

Shipley, W. C. (1940). A self-administered scale for measuring intellectual impairment and deterioration. $J$. Psychol. 9, 371-377.
Smith, S. M. (2002). Fast robust automated brain extraction. Hum. Brain Mapp. 17, 143-155.

Smith, S. M., Jenkinson, M., Woolrich, M. W., Beckmann, C. F., Behrens, T. E., Johansen-Berg, H., Bannister, P. R., De Luca, M., Drobnjak, I., Flitney, D. E., Niazy, R. K., Saunders, J., Vickers, J., Zhang, Y., De Stefano, N., Brady, J. M., and Matthews, P. M. (2004). Advances in functional and structural MR image analysis and implementation as FSL. Neuroimage 23 (Suppl. 1), S208-S219.

Song, S. K., Sun, S. W., Ramsbottom, M. J., Chang, C., Russell, J., and Cross, A. H. (2002). Dysmyelination revealed through MRI as increased radial (but unchanged axial) diffusion of water. Neuroimage 17, 1429-1436.

Sundaram, S. K., Sivaswamy, L., Makki, M. I., Behen, M. E., and Chugani, H. T. (2008). Absence of arcuate fasciculus in children with global developmental delay of unknown etiology: a diffusion tensor imaging study. J. Pediatr. 152, 250-255.

Trainor, L. J., Desjardins, R. N., and Rockel, C. (1999). A comparison of contour and interval processing in musicians and non-musicians using eventrelated potentials. Aust. J. Psychol. 51, 147-153.

Vernooij, M. W., Smits, M., Wielopolski, P. A., Houston, G. C., Krestin, G. P., and Van Der Lugt, A. (2007). Fiber density asymmetry of the arcuate fasciculus in relation to functional hemispheric language lateralization in both rightand left-handed healthy subjects: a combined fMRI and DTI study. Neuroimage 35, 1064-1076.

Wan, C. Y., Rueber, T. R., Hohmann, A., and Schlaug, G. (2010a). The therapeutic effects of singing in neurological disorders. Music Percept. 27, 287-295.

Wan, C. Y., Demaine, K., Zipse, L., and Schlaug, G. (2010b). From music making to speaking: engaging the mirror neuron system in autism. Brain Res. Bull. 82, 161-168.

Wan, C. Y., and Schlaug, G. (2010a) Neural pathways for language in autism: the potential for music-based treatments. Future Neurol. 5, 797-805.

Wan, C. Y., and Schlaug, G. (2010b). Music making as a tool for promoting brain plasticity across the life-span. Neuroscientist 16, 566-577.

Wheeler-Kingshott, C.A., and Cercignani, M. (2009). About "axial" and "radial" diffusivities. Magn. Reson. Med. 61, 1255-1260.

Xue, R., Van Zijl, P. C., Crain, B. J., Solaiyappan, M., and Mori, S. (1999). In vivo three-dimensional reconstruction of rat brain axonal projections by diffusion tensor imaging. Magn. Reson. Med. 42, 1123-1127.

Zarate, J. M., Wood, S., and Zatorre, R. J. (2010). Neural networks involved in voluntary and involuntary vocal pitch regulation in experienced singers. Neuropsychologia 48, 607-618.
Zarate, J. M., and Zatorre, R. J. (2008). Experience-dependent neural substrates involved in vocal pitch regulation during singing. Neuroimage 40, 1871-1887.

Zatorre, R. J., Chen, J. L., and Penhune, V. B. (2007). When the brain plays music: auditory-motor interactions in music perception and production. Nat. Rev. Neurosci. 8, 547-558.

Conflict of Interest Statement: The authors declare that the research was conducted in the absence of any commercial or financial relationships that could be construed as a potential conflict of interest.

Received: 17 March 2011; paper pending published: 16 April 2011; accepted: 23 June 2011; published online: 07 July 2011.

Citation: Halwani GF, Loui P, Rüber T and Schlaug $G$ (2011) Effects of practice and experience on the arcuate fasciculus: comparing singers, instrumentalists, and non-musicians. Front. Psychology 2:156. doi: 10.3389/fpsyg.2011.00156

This article was submitted to Frontiers in Auditory Cognitive Neuroscience, a specialty of Frontiers in Psychology.

Copyright (c) 2011 Halwani, Loui, Rüber and Schlaug. This is an open-access article subject to a non-exclusive license between the authors and Frontiers Media SA, which permits use, distribution and reproduction in other forums, provided the original authors and source are credited and other Frontiers conditions are complied with. 